\title{
CHANNEL GUIDING FOR LASER WAKEFIELD ACCELERATORS*
}

\author{
$\underline{\text { W.P. Leemans }}{ }^{\dagger}$ P. Volfbeyn, L. Archambault, P. Catravas, S. Dimaggio, and E. Esarey \\ Lawrence Berkeley National Laboratory, University of California, Berkeley CA 94720
}

\section{Abstract}

Experimental investigations of laser guiding in plasma channels at LBNL are reported. Intense $\left(>2 \times 10^{17}\right.$ $\mathrm{W} / \mathrm{cm}^{2}$ ), short (60 fs) laser pulses have been injected and guided in slab or cylindrical channels. The channels are produced in $H_{2}$ gas, delivered by a high pressure gasjet, with the dual-pulse Ignitor-Heater technique [1]. Using cylindrical focusing optics, a first ultrashort $(<100 \mathrm{fs})$ laser pulse (Ignitor), is brought to a line focus to ionize the gas jet. A second 160 ps long laser pulse (Heater) is subsequently used to heat the existing spark via inverse Bremsstrahlung. The hydrodynamic shock expansion creates a channel. The channel profile is diagnosed with time resolved longitudinal interferometry. The effects of laser beam size and divergence mismatch at the channel entrance and leakage of the laser energy out of the channel are studied in $1 \mathrm{D}$ and 2D.

\section{INTRODUCTION}

Optical guiding of intense laser pulses in plasma channels [2] is beneficial to a variety of applications, including plasma-based accelerators [3]. The distance over which a laser beam propagates in vacuum is limited by the diffraction or Rayleigh length $Z_{R}=\pi r_{0}^{2} / \lambda$, where $r_{0}$ is the laser spot radius at focus of a Gausssian laser mode with an intensity profile $I \propto \exp \left(-2 r^{2} / r_{0}^{2}\right), \lambda=2 \pi c / \omega_{0}$ is the laser wavelength, and $\omega_{0}$ is the laser frequency. For example, in the laser wakefield accelerator [3], plasma wave excitation requires laser intensities on the order of $10^{18} \mathrm{~W} / \mathrm{cm}^{2}$, which implies $\mu \mathrm{m}$-scale spot sizes for TW-level laser powers $\left(Z_{R} \simeq 300 \mu \mathrm{m}\right.$ for $r_{0}=10 \mu \mathrm{m}$ and $\left.\lambda=1 \mu \mathrm{m}\right)$. Hence, high electron energy gains require guiding over long acceleration distances (many $Z_{R}$ ).

Plasma channels have been proposed as a means of guiding laser pulses $[2,4,6-8]$. The index of refraction in a plasma of density $n$ is approximately $\eta_{R} \simeq 1-\omega_{p}^{2} / 2 \omega_{0}^{2}$, where $\omega_{p}=\left(4 \pi n e^{2} / m_{e}\right)^{1 / 2}$ is the electron plasma frequency. As in an optical fiber, a plasma channel can provide optical guiding if the index of refraction peaks on axis, $\partial \eta_{R} / \partial r<0$, which can be achieved with a plasma density profile that has a local minimum on axis, $\partial n / \partial r>0$. Specifically, a channel with a radially parabolic density profile of the form $n(r)=n_{0}+\Delta n r^{2} / r_{0}^{2}$ can guide a Gaussian laser pulse with a constant spot size $r_{0}$ provided the channel depth $\Delta n$ satisfies $\Delta n=\Delta n_{c}$, where $\Delta n_{c}=1 / \pi r_{e} r_{0}^{2}$ is the critical channel depth [4] and $r_{e}=$

\footnotetext{
* Work supported by the U.S. Department of Energy under contract No. DE-AC-03-76SF0098.

${ }^{\dagger}$ Email: wpleemans@1bl.gov
}

$e^{2} / m_{e} c^{2}$ is the classical electron radius, i.e., $\Delta n_{c}\left[\mathrm{~cm}^{-3}\right] \simeq$ $1.13 \times 10^{20} / r_{0}^{2}[\mu \mathrm{m}]$.

Plasma channels have been created in the laboratory by a variety of methods: (i) Passing a long laser pulse through an optic to create a line focus in a gas, which ionizes and heats the gas, creating a radially expanding hydrodynamic shock [1,5-11], (ii) using a slow capillary discharge to control the plasma profile [12], and (iii) using the ponderomotive force of an intense, relativistically self-guided laser pulse in a plasma, which creates a channel in its wake [1321].

To guide highly intense laser pulses, plasmas channels must be produced in deeply ionized gases, where the density profile cannot be changed by the guided pulse through further ionization. An increase in density on-axis would lead to ionization-induced refraction [22] and hence negate the guiding. Reaching sufficient depth of ionization is most easily satisfied through the use of low atomic number, $Z$, gases. In laser created channels, the use of low Z-gases (e.g., hydrogen or helium) requires that the channel producing laser pulse satisfy two conditions: (1) be sufficiently intense to create free electrons through barrier suppression ionization [23] (typically $>2 \times 10^{14} \mathrm{~W} / \mathrm{cm}^{2}$ ) and (2) be energetic and long (several $100 \mathrm{~mJ}$ 's/pulse in $>100 \mathrm{ps}$ ) but relatively low intensity $\left(<1 \times 10^{13} \mathrm{~W} / \mathrm{cm}^{2}\right)$ to efficiently heat the plasma through inverse Bremsstrahlung heating. Short intense pulses are indeed inefficient at heating plasmas since the collision frequency is reduced for large quiver velocities [24].

To meet these two conditions, we have developed a novel technique which, rather than utilizing a single laser pulse for ionization and heating, makes use of two laser pulses [1]. A sub-ps low energy but intense $\left(>2 \times 10^{14} \mathrm{~W} / \mathrm{cm}^{2}\right)$ "Ignitor" pulse is first used to create the initial spark, followed by a long (tens to hundreds of ps), energetic ( $>100$ $\mathrm{mJ})$ "Heater" pulse. Using the Ignitor-Heater method, the total laser energy required to generate a channel with length $L_{c h}$ is greatly reduced compared to a single pulse. As a practical example, to achieve $>2 \times 10^{14} \mathrm{~W} / \mathrm{cm}^{2}$ in a $100 \mathrm{ps}$ long pulse, focused to a spot size of $5 \mu \mathrm{m} \times 5 \mathrm{~mm}\left(L_{c h}=5\right.$ $\mathrm{mm}$ ) would require $5 \mathrm{~J}$ laser energy compared to $5 \mathrm{~mJ}$ for a 100 fs pulse.

The geometrical shape of the plasma channel crosssection is strongly affected by the initial transverse shape of the hot plasma volume. With the Ignitor-Heater method, control of the initial spark shape is possible by appropriate choice of the propagation geometry of the two pulses. As will be shown, slab-like channels were produced by allowing the Ignitor and Heater beams to copropagate and cylindrically symmetric channels were created by propagating the two beams orthogonally to each other thereby limiting 
the hot plasma region to the intersection volume of the two beams.

In practice, the transverse extent and peak plasma density of the channel are finite. Hence, tunneling of the laser radiation through the channel wall becomes an important loss factor [6]. Similarly to glass fibers, coupling a laser beam into a plasma fiber is subject to losses from mode mismatch and reflections at the entrance of the fiber. While the mode mismatch losses in the two cases are very similar, the nature of the entrance reflection is more complicated in a plasma channel. A gas jet must be used in an evacuated chamber to avoid ionization induced refraction [22] that would occur in a statically filled experimental chamber. This paper addresses guiding loss mechanisms and beam propagation issues, both in transversely elongated (slab) channels and in cylindrically symmetric (round) channels.

\section{CHANNEL PRODUCTION}

The experiments were performed using the multi-TW $\mathrm{Ti}: \mathrm{Al}_{2} \mathrm{O}_{3}$ laser $(\lambda \simeq 820 \mathrm{~nm}$ ) at the l'OASIS laboratory of the Center for Beam Physics at Lawrence Berkeley National Laboratory $[1,10]$. To implement the Ignitor-Heater method, two laser pulses were combined in a line-focus by means of cylindrical optics onto a gas jet (Fig. 1). A short (typically 60-75 fs), 20-40 mJ intense Ignitor pulse was focused to $\mathrm{a} \sim 5 \mu \mathrm{m} \times 5 \mathrm{~mm}$ area by reflecting off a cylindrical reflector, resulting in an intensity of $\sim 5 \times 10^{14} \mathrm{~W} / \mathrm{cm}^{2}$. The cylindrical reflector was a plano-concave $(R=38$ $\mathrm{mm}$ ) cylindrical lens, coated with a dielectric high reflection coating for $45^{\circ}$ angle of incidence. The use of a reflective optic was required to avoid undesirable nonlinear effects that would prevent obtaining a well focused, near diffraction limited beam spot. The Heater pulse $(\sim 270$ $\mathrm{mJ}, 160 \mathrm{ps}$ long) was focused with an F/5 refractive cylindrical lens (focal length $f_{l}=50 \mathrm{~mm}$ ) at the exact location of the Ignitor focus. In addition to the ease of the optical setup, stemming from the fact that the channel forming beams propagate perpendicularly to the guided pulse, the use of two independent cylindrical optics provided precise independent adjustment of the angles of incidence and postions of the line foci.

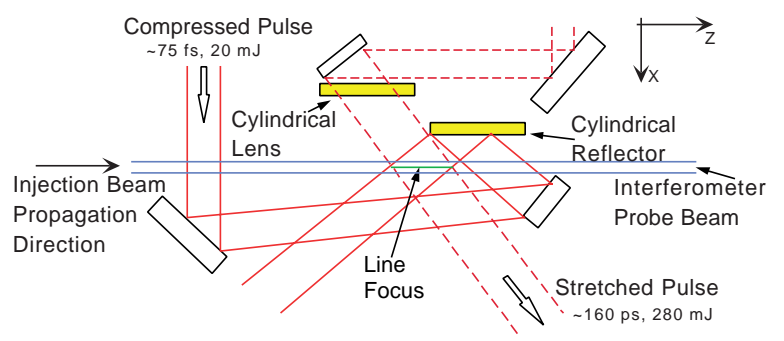

Figure 1: Experimental Setup.

A Mach-Zehnder type interferometer with a measured spatial resolution of $4 \mu \mathrm{m}$ was built to measure line integrated plasma density. This interferometer measures the relative spatial phase shift between two blue $(410 \mathrm{~nm}) \sim$
50 fs pulses, one propagating through plasma (down the channel axis, perpendicular to the Ignitor-Heater pulses) and one through air (see Fig. 1). These pulses were produced by frequency doubling and were synchronized with the high power beams used in the plasma production. The evolution of the transverse plasma density profile was measured with a temporal resolution determined by the duration of the blue pulse.

The Ignitor-Heater scheme was first implemented with both pulses propagating in the same plane through a a nitrogen- or hydrogen-backed gas jet. In this geometry, heat is deposited there where the Ignitor pulse intensity exceeds the ionization threshold. Figure 2 (a) shows interferograms taken with the interferometer pulse delayed by 560 ps and by $1 \mathrm{~ns}$ with respect to the Heater pulse in $\mathrm{N}_{2}$ at 1000 psi. ¿From the inferred plasma density line-outs of Fig. 2 (b), it is seen that a plasma density depression is created only in the vertical direction. These slab channels provide one-dimensional (1D) guiding. The $x$-size of the channels roughly corresponds to the Rayleigh range of the Ignitor pulse. Figure 3 shows, as obtained from interferograms (e.g., Fig. 2), the evolution of (a) maximum density $n_{\max }$, (b) distance between density peaks $2 x_{c h}$, and (c) $\Delta n_{c h}=n_{\max }-n_{0}$ of the channels, where $n_{0}$ is the on-axis density, versus time $T$ after spark ignition in $\mathrm{N}_{2}$ at 1000 psi. As expected from 1D hydrodynamic shock theory [13], $x_{c h} \sim T^{2 / 3}$ and, once plasma production ceases, $n_{\max } \sim T^{-2 / 3}$ (these fits are shown in Fig. 3).
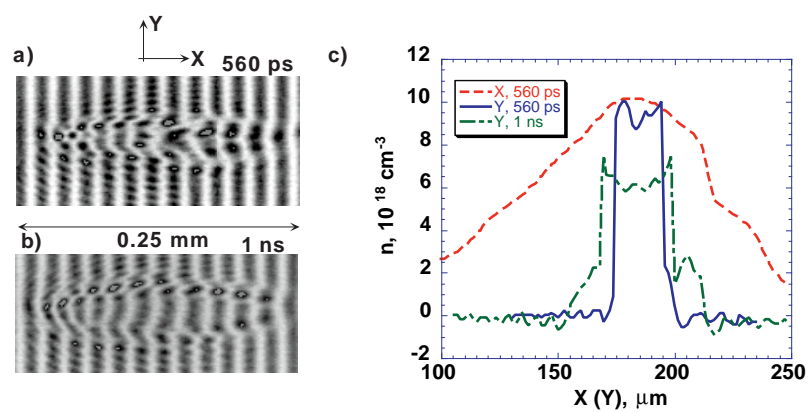

Figure 2: Channel interferograms at (a) 560 ps and (b) $1 \mathrm{~ns}$ after the heater pulse in $\mathrm{N}_{2}$ at 1000 psi, both Ignitor and Heater pulses propagate along the $x$-axis, interferometer beam travels along the $z$-axis, the gasjet plume is much wider than the $x$-extent of the channel; (b) inferred plasma density lineouts: $n(x)$ (dashed curve) and $n(y)$ (solid curve) at $560 \mathrm{ps}$; and $n(y)$ (dot-dash curve) at $1 \mathrm{~ns}$.

To create cylindrically symmetric channels, the Ignitor and Heater beams were made to propagate orthogonally to each other (and to the guided beam). This $90^{\circ}$ geometry limited the transverse extent of the initial heat deposition volume to the small intersection volume of the two beams (typically $5 \mu \mathrm{m} \times 5 \mu \mathrm{m}$ ). Fig. 4 (a) and (b) show the geometry for producing slab-like and cylindrically symmetric plasma channels, respectively.

To demonstrate the change in channel shape, shadow- 

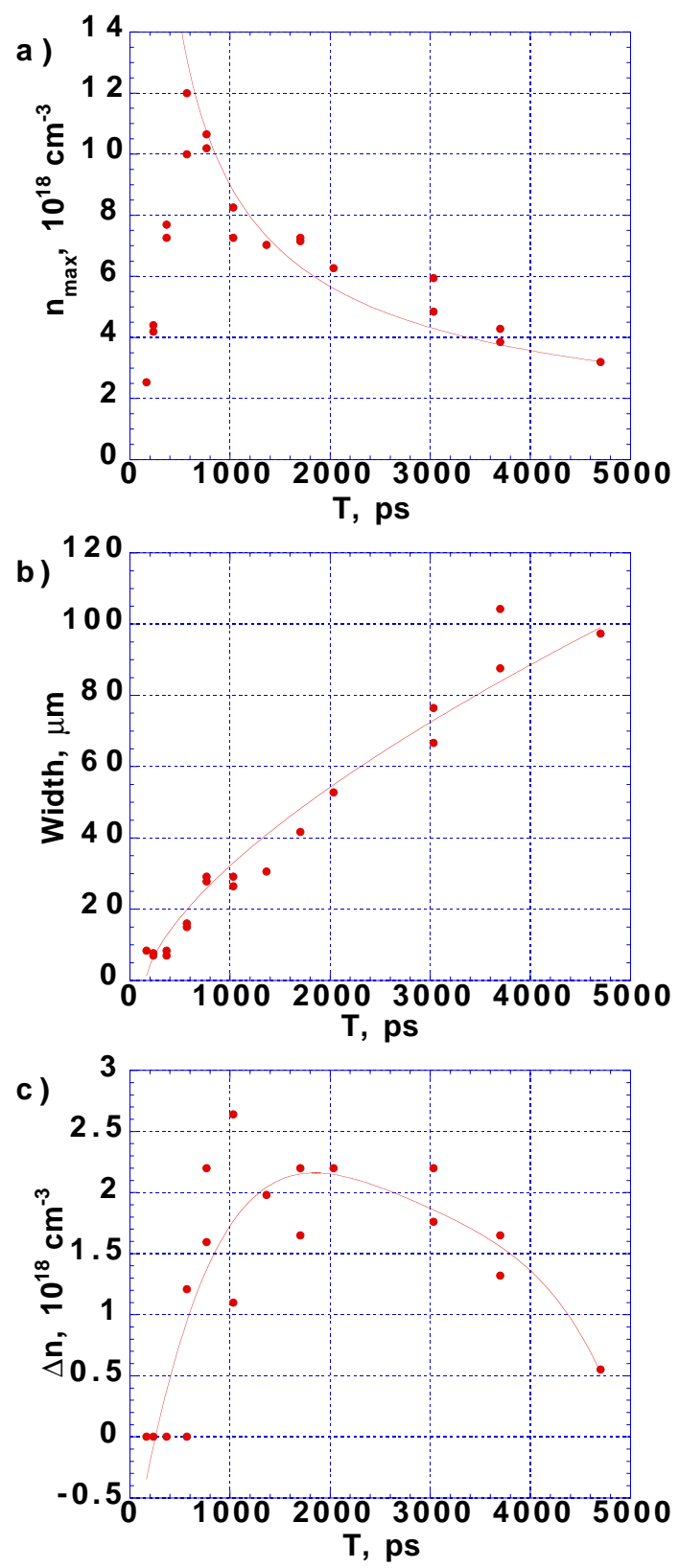

Figure 3: Channel evolution versus time $T$ after spark ignition in $\mathrm{N}_{2}$ at 1000 psi: (a) Maximum density $n_{\max }$ (points are data; curve is a $T^{-2 / 3}$ fit), (b) distance between $n_{\max }$ peaks (points are data; curve is a $T^{2 / 3}$ fit), and (c) $\Delta n_{c h}=n_{\max }-n_{0}$ (points are data; curve is fourth order polynomial fit).

grams were used, Figs. 4 (c) and (d). A shadowgram is obtained by taking an image of the probe interferometric beam (with the reference beam blocked) at the exit of the channel. Refraction in the channel walls expels the blue light, thus casting a shadow on the image of the probe beam. The part of the probe beam that falls inside of the channel walls was observed to be guided. Figures 4 (c) and (d) clearly demonstrate the effect of the geometry change: the $1 \mathrm{D}$ elongated channels of the in-plane configuration,
Fig. 4 (c), are contrasted by the round $90^{\circ}$ configuration channels, Fig. 4 (d).
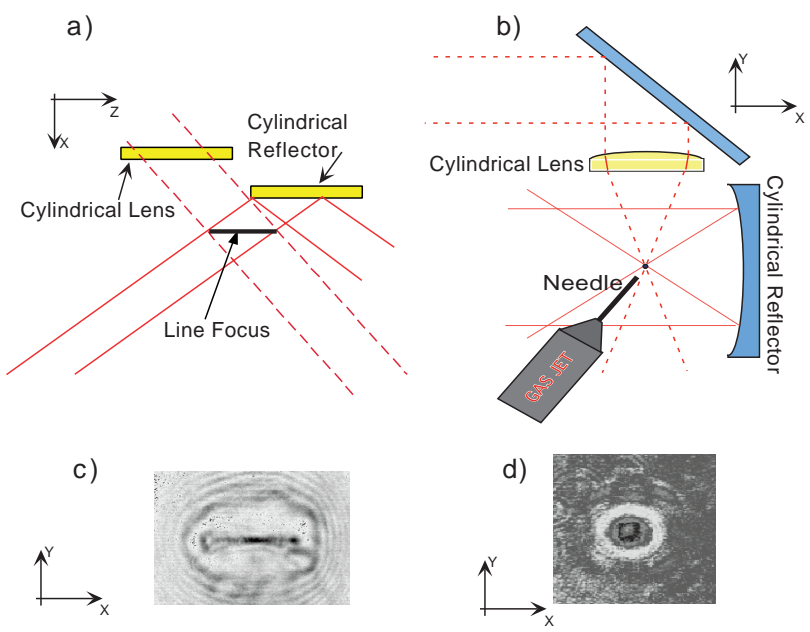

Figure 4: a) In-plane laser beam combining and b) $90^{\circ}$ beam configurations; with c) and d) the corresponding channel shadowgrams in the $x, y$ plane.

\section{GUIDING}

Laser pulses (injection pulses) were focused near the entrance of slab-like and cylindrical channels using an offaxis parabola. To diagnose the guiding, the laser beam was imaged onto a charged-coupled device (CCD) camera using a 1 inch diameter $\mathrm{MgF}_{2}$ lens of focal length of $f_{l}=68.3 \mathrm{~mm}$ at $800 \mathrm{~nm}$. The CCD camera was mounted on an optical rail allowing a change in position of the object plane with a range of $50 \mathrm{~cm}$. The resolution and magnification of the imaging system was calibrated for different CCD camera locations.

Figure 5 shows images of a laser pulse ( $75 \mathrm{fs}, 20-40 \mathrm{~mJ}$, with a minimum focal spot size of $\sim 5 \mu \mathrm{m}$ in vacuum, i.e., $\sim 5 \times 10^{17} \mathrm{~W} / \mathrm{cm}^{2}$ ) injected into the slab-like channels for a) propagating through vacuum (gas jet turned off), b) after undergoing ionization induced refraction [22] in the gas jet plume without the Heater pulse being present, hence no channel formed, and c) guided by the channel, for a gas jet backed with nitrogen at 1000 psi. Vertical line-outs of these images, shown in Fig. 5 d), clearly demonstrate the changes induced by the plasma channel on the guided laser pulse. The guided spot size near the end of the channel was $\sim 8 \mu \mathrm{m} \times 50 \mu \mathrm{m}$. The reduction in vertical beam size by $\sim 8$ times provided by the channel is consistent with a laser beam of $Z_{R} \sim 0.1 \mathrm{~mm}$ propagating a distance of $\sim 0.8 \mathrm{~mm}$ (the half-width of the jet).

As seen in Fig. 2, for the in-plane Ignitor and Heater pulse configuration, plasma channels were created in an elongated, elliptical shape. In turn, the guided beam images (Fig. 5 (c)) had a similar elongated shape.

As the gas jet was moved further away from the laser focus, larger portions of the injected laser energy did not 
a)

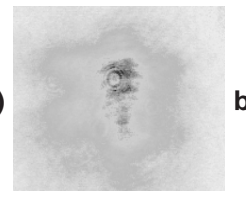

b)

d)

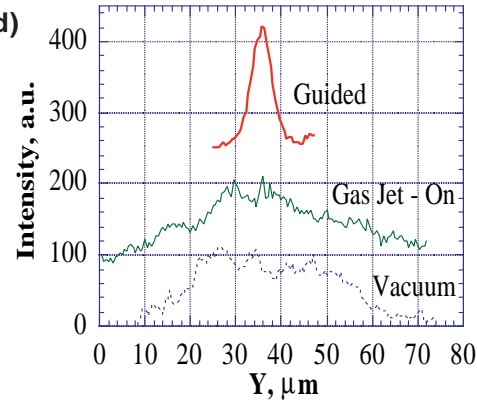

Figure 5: Transverse laser beam images for the beam propagating (a) in vacuum, (b) through the gas jet plume with only the Ignitor pulse, and (c) in a slab-like nitrogen plasma channel. The plane imaged onto the CCD camera was located at the channel exit. The gas jet backing pressure was 1000 psi.

couple into the channel. To obtain a quantitative measurement for the guiding properties of these channels, the image intensity integral of the CCD images was used as a measure of the laser energy. This allowed a comparison of the amount of the laser energy guided by the channel to the total intensity integral of the CCD image (full beam energy, $\left.E_{\text {full }}\right)$. The intensity integral of the isolated central lobe of the images was taken to be the guided energy.

The ratio of the guided energy, $E_{g}$, to the total image intensity integral, $E_{\text {full }}$ is shown in Fig. 6. As expected, the fraction of the laser beam that is coupled into the channel becomes larger as the injection pulse focus is moved closer to the jet's edge than when the gas jet is moved further away from the laser focus position in vacuum. It should be noted that the F-numbers of the off-axis parabola and the imaging $\mathrm{MgF}_{2}$ lens are rather close and the collection angle is limited. Nevertheless, the leakage of the fundamental mode of the plasma channel is fully collected.

As the laser waist is moved closer to the channel entrance, the laser spot size becomes comparable with the channel size and most of the power is coupled to the modes of the channel. The ratio of $E_{g}$ to the $E_{f u l l}$ approaches $\sim 55 \%$. This number is in good agreement with analytical results [1], where the leakage fraction was estimated to be $\sim 50 \%$.

To demonstrate the dependence of the number of guided modes on the channel depth, shadowgrams of the round channels $\left(90^{\circ}\right.$ geometry in $\left.\mathrm{H}_{2}\right)$ were taken with variable time delay with respect to the arrival time of the Heater pulse. As the shock wave propagates further out, leaving a deeper plasma density depression on the channel axis, the guided part of the probe beam couples to higher and higher order modes of the channel. Figure 7 presents the results of

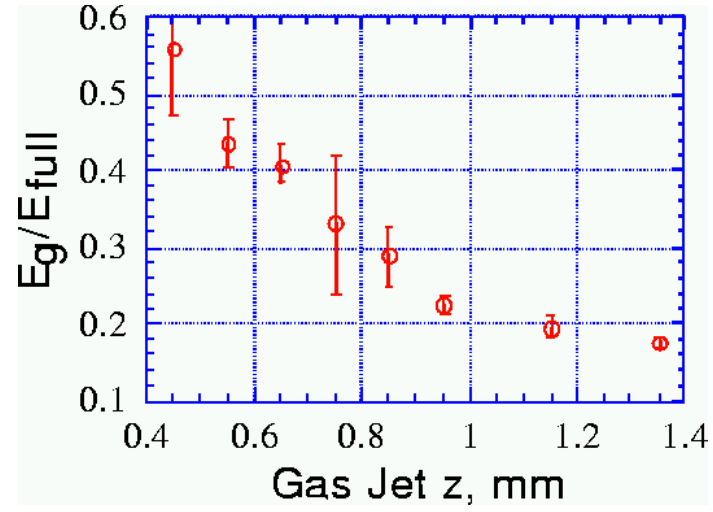

Figure 6: Ratio of energy in the guided central lobe, $E_{g}$, to that of the full beam, $E_{f u l l}$, plotted versus the gas jet position, $z$, where $z=0$ corresponds to the injection pulse focus being in the center of the gas jet plume (half-width of the gas jet plume is $\sim 0.8-1 \mathrm{~mm}$ ).

such a time delay scan taken with a channel created in hydrogen. The increase in the number of lobes in the guided part of the blue pulse for longer probe pulse delays demonstrates the dependence of the number of guided modes on the channel depth and width. Similar measurements were previously reported for channels created in high $Z$ gases $[6,7]$.

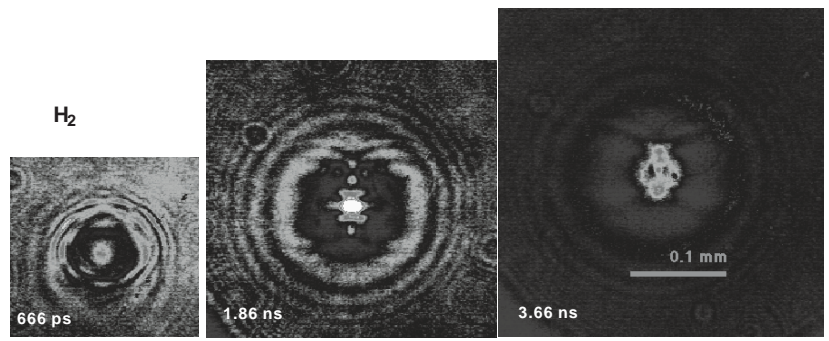

Figure 7: Shadowgrams of round channels created in hydrogen at three different time delays after the heating: 666 ps (left), $1.86 \mathrm{~ns}$ (center), and $3.66 \mathrm{~ns}$ (right).

Beam images of laser pulses $(20 \mathrm{~mJ}$ in $60 \mathrm{fs})$ in vacuum and injected in round channels, produced in a $\mathrm{H}_{2}$ gasjet, are shown in Fig. 8 (a) and (b) respectively. Lineprofiles are shown Fig.8(c). The injected spot size was measured to be about $10 \mu \mathrm{m}$ resulting in an injected intensity of $\sim 2 \times 10^{17}$ $\mathrm{W} / \mathrm{cm}^{2}$. The channeling efficiency was measured to be typically $>70 \%$. Optimization of channeling efficiency and detailed measurements of mode matching and wakefield excitation for higher intensity pulses $\left(\sim 10^{18} \mathrm{~W} / \mathrm{cm}^{2}\right)$ in these channels are underway.

\section{DISCUSSION}

To overcome the laser diffraction length limit, a novel method of plasma channel production for laser guiding, the Ignitor-Heater technique, was proposed and tested experimentally. With this technique, plasma channels can be cre- 
(a)
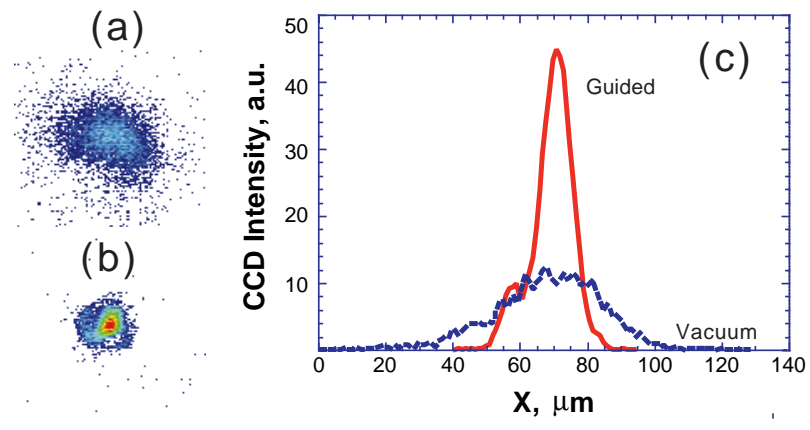

Figure 8: Transverse laser beam images for the beam propagating (a) in vacuum, (b) in a cylindrical hydrogen plasma channel, and (c) the corresponding lineprofiles. The plane imaged onto the CCD camera was located at the channel exit. The gas jet backing pressure was 1000 psi.

ated in hydrogen and deeply ionized nitrogen without high atomic number additives, thereby allowing high intensity laser pulse guiding. To avoid ionization induced refraction of the guided laser pulse, channels were formed in the plume of a pulsed gas jet. It should also be noted that the Ignitor-Heater scheme employs cylindrical optics that are out of the path of the accelerator beam (for plasma-based accelerator applications) and, potentially, may allow the recycling of the laser beams. The channel formation process was fully characterized with time resolved 2D longitudinal interferometry diagnostic using a sub-ps probe pulse. The shape of the initial spark was proven to affect the shape of the plasma channel. By using in-plane or orthogonal beam combining configurations, elongated, or slab channels, with $1 \mathrm{D}$ guiding properties and cylindrically symmetric, round, channels with guiding in both transverse dimensions were created in both nitrogen and hydrogen.

Laser pulses at high intensity $\left(\sim 2-5 \times 10^{17} \mathrm{~W} / \mathrm{cm}^{2}\right)$ were injected in nitrogen slab channels and in hydrogen cylindrical channels and observed to be guided over $\sim$ $5-10$ Rayleigh lengths, limited by the gasjet length. Leakage and transmission measurements for the slab channels were found to agree well with the theoretical predictions. Similar measurements in cylindrical channels are underway.

Single mode and multimode laser propagation in round channels was observed as a function of time delay of the probe pulse injection after the spark heating. The modal content is found to be in good qualitative agreement with predictions from a 2D leakage theory [1].

An optical diagnostic is currently being designed to diagnose the spatial density profile of the channel, and to measure the laser excited wakefields in the channel [25]. Efforts are also directed towards the development of long scalelength high pressure gasjets $(\sim 1-2 \mathrm{~cm})$, resulting in a substantial increase in guiding distance. Such gasjets could also have applications in the field of high harmonic generation [26] and $\mathrm{x}$-ray lasers [27].

\section{ACKNOWLEDGMENTS}

The authors acknowledge useful conversations with S. Chattopadhyay, C.B. Schroeder, B.A. Shadwick, and J.S. Wurtele,the technical support from J. Dougherty and laser safety assistance from $\mathrm{K}$. Barat.

\section{REFERENCES}

[1] P. Volfbeyn and W.P. Leemans, in Proc. 6th European Part. Accl. Conf., Ed. by S. Myers et al., (Inst. of Phys., Philadelphia, 1998), p. 265; P. Volfbeyn, MIT Ph.D. Thesis, LBNL Report 41892 (1998); P. Volfbeyn, E. Esarey and W.P. Leemans, Phys. Plasmas, May (1999).

[2] For a review see, E. Esarey et al., IEEE J. Quantum Electron. 33, 1879 (1997).

[3] T. Tajima and J.M. Dawson, Phys. Rev. Lett. 43, 267 (1979); For a review see, E. Esarey et al., IEEE Trans. Plasma Sci. 24, 252 (1996);

[4] P. Sprangle and E. Esarey, Phys. Fluids B 4, 2241 (1992); P. Sprangle et al., Phys. Rev. Lett. 69, 2200 (1992). W.P. Leemans et al., IEEE Trans. Plasma Sci. 24, 331 (1996).

[5] L.C. Johnson and T.K. Chu, Phys. Rev. Lett. 32, 517 (1974).

[6] C.G. Durfee III and H. M. Milchberg, Phys. Rev. Lett. 71, 2409 (1993); H.M. Milchberg et al., Phys. Plasmas 3, 2149 (1996).

[7] T.R. Clark and H.M. Milchberg, Phys. Rev. Lett. 78, 2373 (1997); T.R. Clark and H.M. Milchberg, Phys. Rev. Lett. 81, 357 (1998).

[8] V. Malka et al., Phys. Rev. Lett. 79, 2979 (1997).

[9] T. Ditmire et al., Opt. Lett. 23, 322 (1998).

[10] W.P. Leemans et al., Phys. Plasmas, 5, 1615 (1998).

[11] E. Gaul et al., Advanced Accelerator Concepts, Edited by W. Lawson, AIP Conf. Proc. (AIP, NY, in press).

[12] Y. Ehrlich et al., Phys. Rev. Lett. 77, 4186 (1996); Y. Ehrlich et al., J. Opt. Soc. Am. B 15, 2416 (1998).

[13] K. Krushelnick et al., Phys. Rev. Lett. 78, 4047 (1997); A. Ting et al., Phys. Plasmas 4, 1889 (1997),

[14] S.Y. Chen et al., Phys. Rev. Lett. 80, 2610 (1998).

[15] A.B. Borisov et al., J. Opt. Soc. Am. B 11, 1941 (1994).

[16] P. Gibbon et al., IEEE Trans. Plasma Sci. 24, 343 (1996).

[17] A. Chiron et al., Phys. Plasmas 3, 1373 (1996).

[18] P.E. Young and P.R. Bolton, Phys. Rev. Lett. 77, 4556 (1996).

[19] M. Borghesi et al., Phys. Rev. Lett. 78, 879 (1997).

[20] J. Fuchs et al., Phys. Rev. Lett. 80, 1658 (1998).

[21] C.E. Clayton et al., Phys. Rev. Lett. 81, 100 (1998).

[22] W.P. Leemans et al., Phys. Rev. A 46, 1091 (1992).

[23] S. Augst et al., Phys. Rev. Lett. 63, 2212 (1989).

[24] C.D. Decker et al., Phys. Plasmas 1, 4043 (1994); G. Shvets and N.J. Fisch, Phys. Plasmas 4, 428 (1997).

[25] ] P. Volfbeyn and W.P. Leemans, these Proceedings.

[26] C.G. Durfee et al., Opt. Lett. 22, 1565 (1997).

[27] N.H. Burnett and P.B. Corkum, J. Opt. Soc. Am. B 6, 1195 (1989); D.C. Eder et al., Phys. Plasmas 1, 1744 (1994). 